\title{
A finite element analysis of the distribution velocity in viscous incompressible fluids using the Lagrange interpolation function
}

\author{
${ }^{1 *}$ AKPOBI, J A; ${ }^{2}$ AKPOBI, E D \\ ${ }^{1}$ Production Engineering Department, University of Benin, Benin City, Nigeria. \\ ${ }^{2}$ Petroleum Engineering Department, University of Benin, Benin City, Nigeria.
}

\begin{abstract}
In this work we use the finite element method to analyze the distribution of velocity in a viscous incompressible fluid flow using Lagrange interpolation function. The results obtained are highly accurate and converge fast to the exact solution as the number of elements increase. @JASEM
\end{abstract}

A fluid is basically any substance that deforms continuously. Its motion is governed by the Stokes equations also known as Navier-Stokes equations. The fluid may be compressible or incompressible. In this study, we consider viscous incompressible fluids. It should be noted that stokes equation can also be used to model turbulent flow, in this case, the fluid parameters are treated as time average values (Lions, 1998 and Efunda, 2005). Finite element method is well treated in many standard texts (Burnett, 1987; Bickford, 1989; and Fagan, 1992). The determination of an accurate characterization of fluid flow has been the subject of much research. Some of these researchers include Stokes, Reynolds, Navier, Bernoulli, Petroff, Sommerfeld etc. Their works formed the fundamental principles of fluid dynamics. (Fox; McDonald,1996; Massey; Wardsmith, 2001)

Also, Demkowicz et. al. (1990) developed a new finite element method for solving compressible
Navier-Stokes equations. Their method was based on a version of Strang's operator splitting and an $h-p$ adaptive finite element approximation in space.

$\mathrm{Wu}$ and Jin (2005) proposed a numerical method for solving Stokes equation with corner singularity. First, they solved a simple eigenvalue problem, which was one dimension less than the original problem, to obtain the discrete expansion of the singularity near the corner. And then combined the approximation of the singularity and standard finite element basis functions to construct special finite element spaces, and solve the original problem in the special spaces on a conventional mesh.

In this work we use the finite element method to analyze the distribution of velocity in a viscous incompressible fluid flow using Lagrange interpolation function.

\section{MATHEMATICAL FORMULATION}

Consider the relation:

$$
\frac{\partial p}{\partial t}+\frac{\partial}{\partial x}(\rho u)+\frac{\partial}{\partial y}(\rho u)=0
$$

At steady state, $\quad \frac{\partial}{\partial t}=0$

Thus, we have:

$$
\frac{\partial}{\partial x}(\rho u)+\frac{\partial}{\partial y}(\rho u)
$$

for incompressible fluids, $\rho=$ constant ( $\rho$ is density ), this implies that:

$\rho\left[\frac{\partial u}{\partial x}+\frac{\partial v}{\partial y}\right]=0$

from the continuity expression,

$$
\left[\frac{\partial u}{\partial x}+\frac{\partial v}{\partial y}\right]=0
$$

*Corresponding author 
from the conservation of linear momentum, for incompressible fluid flow we have:

$$
\begin{aligned}
& \nabla \sigma+f=\rho\left[\frac{\partial u}{\partial t}+u \cdot \nabla u\right] \\
& \frac{\partial}{\partial x} \sigma_{x}+\frac{\partial \sigma_{x y}}{\partial y} f_{x}=\rho\left[\frac{\partial u}{\partial t}+u \frac{\partial u}{\partial t}+v \frac{\partial u}{\partial y}\right] \\
& \frac{\partial}{\partial x} \sigma_{x y}+\frac{\partial \sigma_{y}}{\partial y}+f_{y}=\rho\left[\frac{\partial v}{\partial t}+u \frac{\partial v}{\partial x}+v \frac{\partial u}{\partial x}\right] \\
& \sigma_{x}=\tau_{x}-p \\
& \sigma_{y}+p=\tau_{y}
\end{aligned}
$$

where $\sigma_{x}, \sigma_{y}, \sigma_{x y}$ are stress components, $t$ is Time, $u$ is the velocity and $\mu$ is the viscosity . $\tau_{x}, \tau_{y}, \tau_{x y}$ are viscous stress components, while $p$ is the hydrostatic pressure

$\tau_{x}=2 \mu \frac{\partial u}{\partial x}, \tau_{y}=2 \mu \frac{\partial v}{\partial x}, \tau_{x y}=\mu\left(\frac{\partial u}{\partial x}+\frac{\partial u}{\partial x}\right)$

by substitution,

$$
\begin{aligned}
& \frac{\partial}{\partial x}\left(\tau_{x}-p\right)+\frac{\partial}{\partial y}\left(\tau_{x y}\right)+f_{x}=\rho\left(\frac{\partial v}{\partial t}+u \frac{\partial u}{\partial x}+v \frac{\partial v}{\partial y}\right) \\
& \frac{\partial}{\partial x}\left(\tau_{x y}\right)+\frac{\partial}{\partial y}\left(\tau_{y}-p\right)+f_{y}=\rho\left(\frac{\partial v}{\partial t}+u \frac{\partial u}{\partial x}+v \frac{\partial v}{\partial y}\right)
\end{aligned}
$$

rearranging, since

$$
\frac{\partial u}{\partial x}+\frac{\partial v}{\partial y}=0
$$

then, $\frac{\partial}{\partial x}\left(2 \mu \frac{\partial u}{\partial x}-P\right)+\frac{\partial}{\partial y}\left(\mu\left(\frac{\partial u}{\partial y}+\frac{\partial v}{\partial x}\right)\right)+f_{x}=\rho \frac{\partial u}{\partial t}$

$\frac{\partial}{\partial x}\left(\mu\left(\frac{\partial u}{\partial y}+\frac{\partial v}{\partial x}\right)\right)+\frac{\partial}{\partial y}\left(\mu\left(\frac{\partial u}{\partial y}-P\right)\right)++f_{y}=\rho \frac{\partial v}{\partial t}$

Restricting the Stoke's equation for viscous, incompressible fluid flow, we have:

let $f_{x}=0, P=P(x), u=u(y), v=0$

for steady state condition, $\frac{\partial}{\partial t}=0$

thus,

$-\frac{\partial}{\partial x} P(x)+\frac{\partial}{\partial y}\left(\mu \frac{\partial u(y)}{\partial y}\right)=0$

hence,

$\mu \frac{\partial^{2} u(y)}{\partial y^{2}}=\frac{\partial P(x)}{\partial x}$ 
this is the reduced Stoke's model for viscous, incompressible, steady flow.

Now considering equation. 14

$\frac{\partial}{\partial x}\left(\partial u \frac{\partial u}{\partial x}-P\right)+\frac{\partial}{\partial y}\left(\mu\left(\frac{\partial v}{\partial y}+\frac{\partial v}{\partial x}\right)\right)+f_{x}=\rho \frac{\partial u}{\partial t}$

Let $f_{x}=0, P=P(x), u=u(y), v=0$

For steady state condition $\frac{\partial}{\partial t}=0$

thus, $\quad-\frac{\partial}{\partial x}(P(x))+\frac{\partial}{\partial y} \mu\left(\frac{\partial u(y)}{\partial y}\right)=0$

hence $\quad \mu \frac{\partial^{2} u(y)}{\partial y^{2}}=\frac{\partial P(x)}{\partial x}$

thus the stoke's model for viscous, incompressible, steady flow is

$\mu \frac{\partial^{2} u(y)}{\partial y^{2}}=\frac{\partial P(x)}{\partial x}$

Developing the Velocity Distribution Model for Boundary Value Flow Problems

Since the boundary value problem are described by:

$-\frac{d}{d y}\left(a \frac{d u}{d y}\right)+c u-q=0$ for $0<x<l$

comparing this with the developed expression for steady state, viscous incompressible fluid flow

$\mu \frac{\partial^{2} u(y)}{\partial y^{2}}=\frac{\partial P(x)}{\partial x}$

rearranging and comparing:

$-\frac{d}{d y}\left(a \frac{d u}{d y}\right)+c u-q=0$

$\mu \frac{\partial^{2} u(y)}{\partial y^{2}}-\frac{\partial P(x)}{\partial x}=0$

we have $\quad a=\mu, c=0, q=\frac{\partial P(x)}{\partial x}$.

Generating a finite element model for boundary value problems:

$-\frac{d}{d y}\left(a \frac{d u}{d y}\right)+c u-q=0$ for $0<x<l$

where $a=a(y), c=c(y), q=q(y), u_{0}$ and $Q_{0}$ are defined

obtaining the weak form:

$-w\left[\frac{d}{d y}\left(a \frac{d u}{d y}\right)\right]=-\frac{d}{d y}\left(w a \frac{d u}{d y}\right)+a \frac{d w}{d y} \cdot \frac{d u}{d y}$

Integrating over the element domain:

AKPOBI, $J$ A; AKPOBI, E D 
$-\int_{y_{b}}^{y_{a}} w\left(\frac{d}{d y}\left(a \frac{d u}{d y}\right)\right) d y=-\int_{y_{b}}^{y_{a}}\left(w a \frac{d u}{d y}\right) d x ?+\int_{y_{b}}^{y_{a}} a \frac{d w}{d y} \frac{d u}{d y} d y$

$-\int_{y_{A}}^{y_{B}} w\left(\frac{d}{d y}\left(a \frac{d u}{d y}\right)\right) d y=-\left[w a \frac{d u}{d y}\right]_{y_{A}}^{y_{B}}+\int_{y_{b}}^{y_{a}} a \frac{d w}{d y} \frac{d u}{d y} d y$

but $\int_{y_{A}}^{y_{B}} w\left(-\frac{d}{d y}\left(a \frac{d u}{d y}\right)+c u-q\right) d y=0$

substituting into equation. (20), we have:

$\int_{y_{A}}^{y_{B}}\left(\frac{d}{d y}\left(a \frac{d u}{d y}\right)+c w u-w q\right) d y-\left[w a \frac{d u}{d y}\right]_{y_{A}}^{y_{B}}=0$

obtaining the primary and secondary variable from the weak form:

$a \frac{d u}{d y} \quad$ secondary variable

$u$ primary variable

boundary condition assumption:

$-Q_{A}=\left(a \frac{d u}{d y}\right)_{y_{A}}$

$Q_{B}=\left(a \frac{d u}{d y}\right)_{y_{B}}$

thus the variational form is:

$\int_{y_{A}}^{y_{B}}\left(\frac{d}{d y}\left(a \frac{d u}{d y}\right)+c w u-w q\right) d y-w a\left(y_{A}\right) Q_{A}-w a\left(y_{B}\right) Q_{B}$

For an arbitrary degree interpolation

$u \approx u^{e}=\sum_{j=1}^{n} u_{j}^{e} \psi_{j}^{e}(y)$

where $\psi_{j}^{e}(y)$ are the langrage interpolation of $(n-1)$ degree . Rearranging we have:

$\int_{y_{A}}^{y_{B}}\left(\frac{d}{d y}\left(a \frac{d u}{d y}\right)+c w u\right) d y-\int_{y_{A}}^{y_{B}} w q d y-\sum_{i=1}^{n} w y_{i}^{e} Q_{i}^{e}$

substituting

$u^{e}=\sum_{j=1}^{n} u_{j}^{e} \psi_{j}^{e}(y)$ for $u$ and $\psi_{1}^{e}, \psi_{2}^{e}, \psi_{n}^{e}$ for $\mathrm{w}$ for ith equation

$0=\int_{y_{A}}^{y_{B}}\left[a \frac{d \psi_{i}}{d y}\left(\sum_{j=1}^{n} u_{j} \frac{d \psi_{j}^{e}}{d y}\right)+c \psi_{i}^{e}\left(\sum_{i=1}^{n} u_{j} \psi_{j}^{e}(y)-\psi_{j}^{e} q\right)\right] d y-\sum_{j=1}^{n} \psi_{i}^{e} y_{i}^{e} Q_{i}^{e}$

thus obtaining a general term, 
$0=\int_{y_{A}}^{y_{B}}\left(a \frac{d \psi_{n}}{d y}\left(\sum_{j=1}^{n} u_{j} Q_{i}^{e} \frac{d \psi_{j}^{e}}{d y}\right)+c \psi_{n}^{e}\left(\sum_{i=1}^{n} u_{j}^{e} \psi_{j}^{e} c_{y}\right)-\psi_{j}^{e} q\right) d y-\sum_{i=1}^{n} \psi_{j}^{e} y_{i}^{e} Q_{i}^{e}$

this could be given as:

$0=\sum_{j=1}^{n} k_{i j}^{e} \psi_{j}^{e} u_{j}^{e}-f_{i}^{e}-Q_{i}^{e}$

thus

$k_{i j}^{e}=\int_{y_{A}}^{y_{B}}\left(a \frac{d \psi_{i}}{d y} \frac{d \psi_{i}}{d y}+c \psi_{i}^{e} \psi_{j}\right) d y$

$f_{i}=\int_{y_{A}}^{y_{B}} q \psi_{i}^{e} d y$

$Q_{i}^{e}=\sum_{i=1}^{n} \psi_{j}^{e} y_{i}^{e} Q_{i}^{e}$

In matrix form we have

$\left[k^{e}\right]\left\{u^{e}\right\}=\left\{f^{e}\right\}+\left\{Q^{e}\right\}$

Considering the governing equation for the viscous incompressible flow

$\mu \frac{\partial^{2} u(y)}{\partial y^{2}}=\frac{\partial P(x)}{\partial x}$

from its comparison with the standard equation of a boundary value problem we have:

$a=\mu, c=0, q=\frac{\partial P(x)}{\partial x}$

Numerical Examples: To illustrate the model and its accuracy, we consider the following Examples.

Example 1: Consider a parallel flow between two flat plates separated by a distance of $80 \mathrm{~m}$. Determine the velocity distribution for a velocity gradient of $5 \mathrm{~N} / \mathrm{m} 3$ and fluid viscosity of $u=0.8 \mathrm{Kg} / \mathrm{m} / \mathrm{s}$, when he velocity at the end of the plates is zero.

Solution: Interpolation method: Lagrange Interpolation technique. Element type: Linear. The solutions obtained using the model are shown in Fig. 1. 


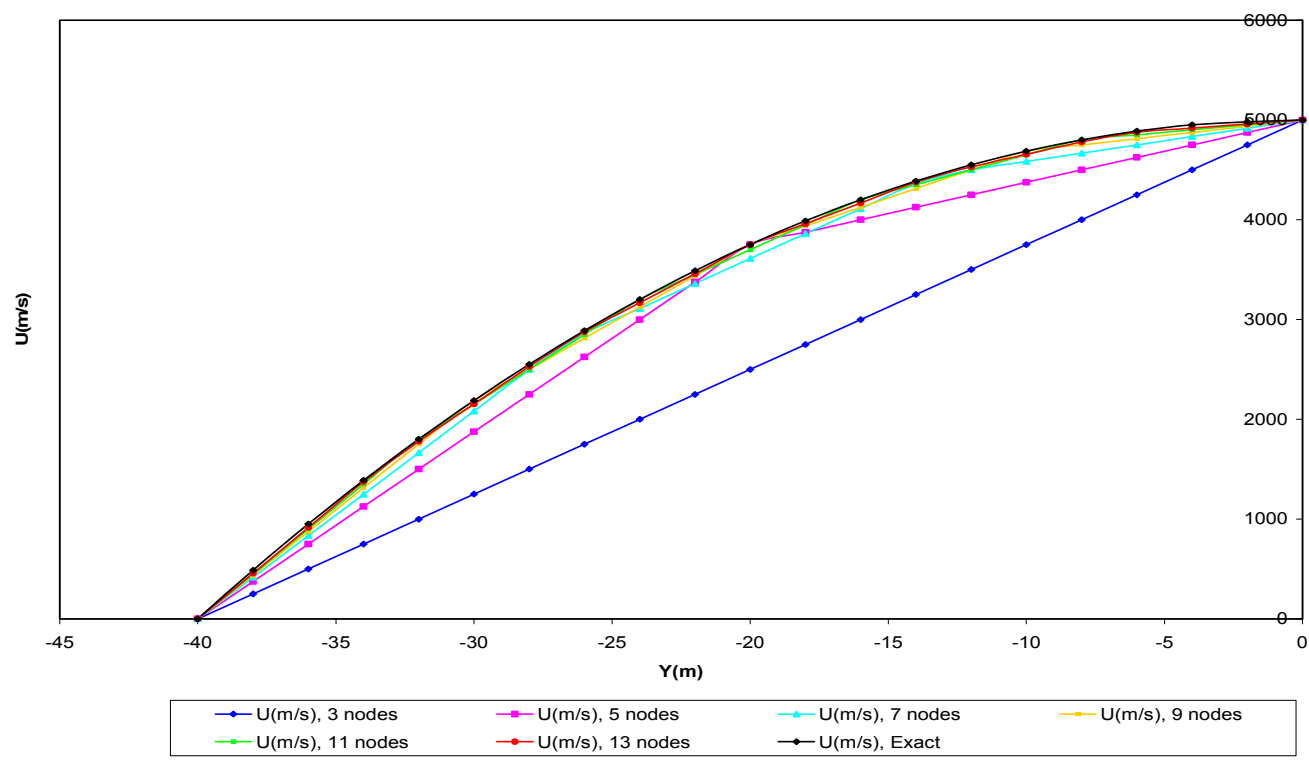

Fig.1: A graph of velocity against displacement for 2, 4, 6, 8, 10, 12 elements and the exact.

Example 2: A viscous, incompressible fluid flows between two flat and parallel plates which are $100 \mathrm{~m}$ apart and at a constant pressure gradient of $12 \mathrm{~N} / \mathrm{m} 3$ and he fluid is of viscosity of $0.92 \mathrm{~kg} / \mathrm{m} / \mathrm{s}$. determine its velocity distribution $U(y)$ if the velocity at the wall of the plates are $U=0 \mathrm{~m} / \mathrm{s} ; U_{b}=400 \mathrm{~m} / \mathrm{s}$

Solution: Interpolation method: Lagrange interpolation technique. Element type: Linear. The solutions obtained using the model are shown in Fig. 2.

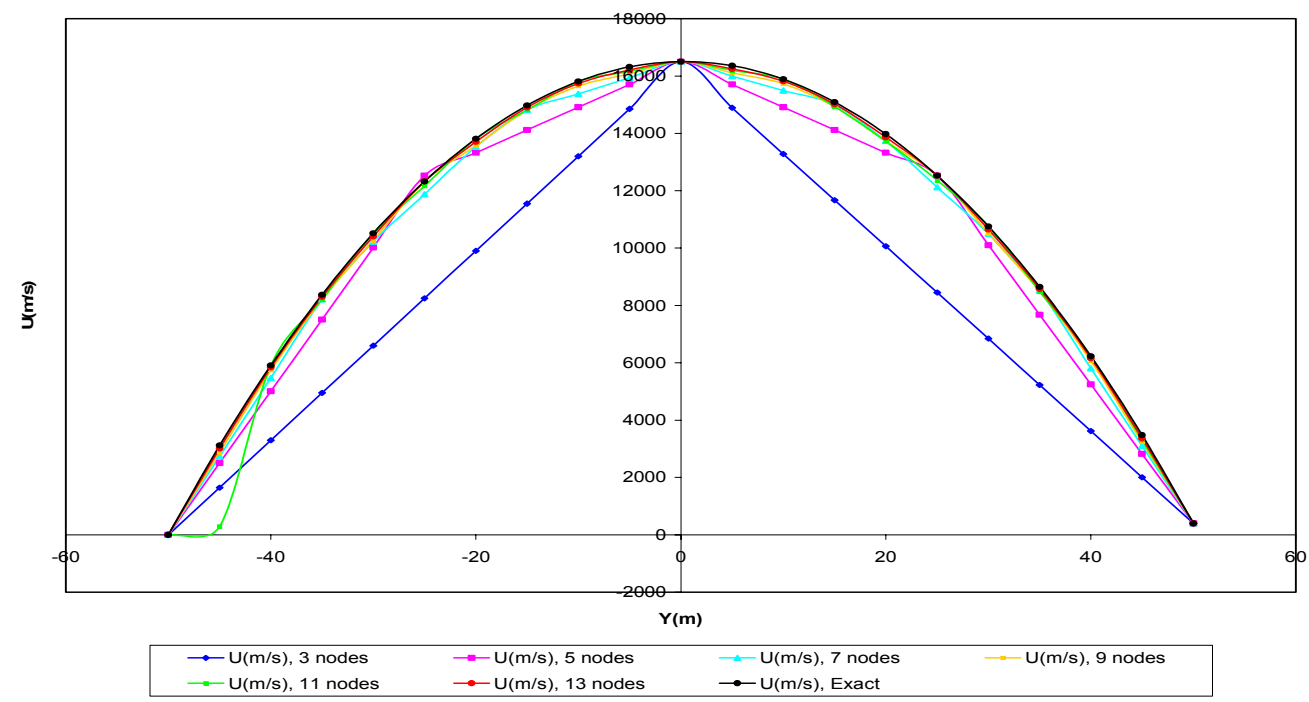

Fig. 2: A graph of velocity against displacement for 2, 4, 6, 8, 10, 12 elements and exact solution 
Example 3: Using the finite element model determine the velocity distribution $\mathrm{U}(\mathrm{y})$ of the flow of a viscous, incompressible fluid flowing through two flat and long walls that are $60 \mathrm{~m}$ apart and has a constant pressure gradient $\frac{d p}{d x}$ of about $20 \mathrm{~N} / \mathrm{m} 3$ if the viscosity of the viscous incompressible fluid is $0.4 \mathrm{~kg} / \mathrm{m} / \mathrm{s}$

Note: both parallel plates have zero velocity

Solution: Interpolation method: Lagrange Interpolation technique. Element type: Linear. The solutions obtained using the model are shown in Fig. 3.

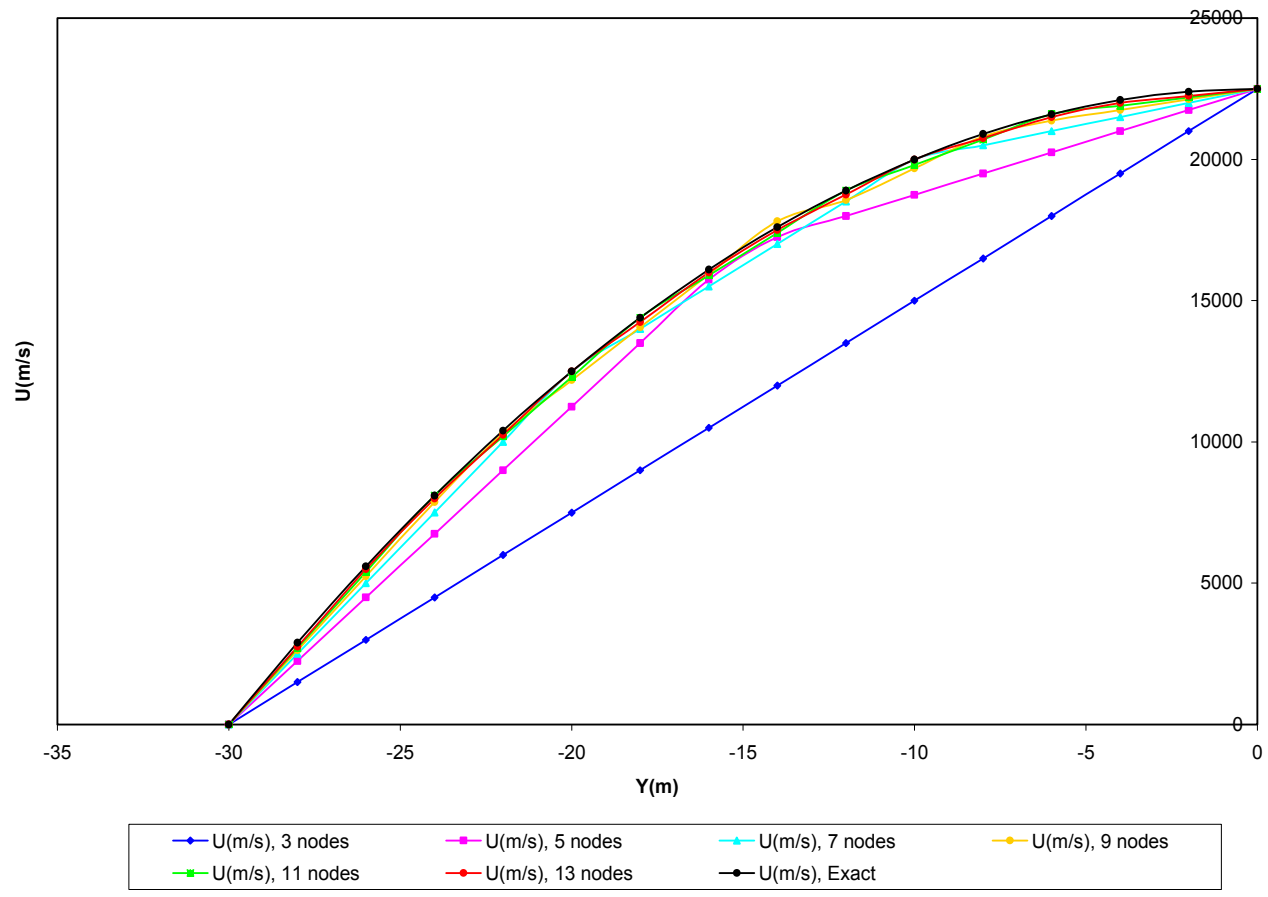

Fig. 3: A graph of velocity against displacement for 2, 4, 6, 8, 10, 12 elements, and the exact

Solution

Table1. Percentage difference of values between analytical solutions and solutions from our mode for all the three Examples

\begin{tabular}{llll}
\hline Node & Example & Example & Example \\
1 & 1 & 2 & 3 \\
2 & $0.00 \%$ & $0.00 \%$ & $0.00 \%$ \\
3 & $0.44 \%$ & $0.67 \%$ & $0.67 \%$ \\
4 & $0.65 \%$ & $0.46 \%$ & $0.45 \%$ \\
5 & $0.23 \%$ & $0.48 \%$ & $0.46 \%$ \\
6 & $0.44 \%$ & $0.79 \%$ & $0.72 \%$ \\
7 & $0.72 \%$ & $0.01 \%$ & $0.00 \%$ \\
\hline
\end{tabular}

AKPOBI, $J$ A; AKPOBI, ED

\begin{tabular}{llll}
\hline 8 & $0.27 \%$ & $0.86 \%$ & $0.57 \%$ \\
9 & $0.78 \%$ & $1.22 \%$ & $0.62 \%$ \\
10 & $0.71 \%$ & $0.28 \%$ & $1.04 \%$ \\
11 & $0.00 \%$ & $0.00 \%$ & $0.00 \%$ \\
12 & $0.82 \%$ & $0.66 \%$ & $1.44 \%$ \\
13 & $1.02 \%$ & $0.46 \%$ & $1.23 \%$ \\
14 & $0.41 \%$ & $0.48 \%$ & $0.79 \%$ \\
15 & $0.86 \%$ & $0.78 \%$ & $1.17 \%$ \\
16 & $1.57 \%$ & $0.09 \%$ & $0.00 \%$ \\
17 & $1.21 \%$ & $1.01 \%$ & $0.62 \%$ \\
18 & $0.93 \%$ & $0.84 \%$ & $1.04 \%$ \\
\hline
\end{tabular}




\begin{tabular}{llll}
\hline 19 & $0.38 \%$ & $1.16 \%$ & $0.00 \%$ \\
20 & $0.91 \%$ & $0.24 \%$ & $0.48 \%$ \\
21 & $0.00 \%$ & $0.00 \%$ & $0.02 \%$ \\
\hline
\end{tabular}

\section{DISCUSSION}

To illustrate the use and accuracy of the model developed, 3 Examples were considered and the solution are shown in Figures 1-3. The solutions to Example 1 are shown in Fig.1. For Example 2, the solutions are shown in Figures 2, while Fig.3 represents the solutions to Example 3.

In Example 1, Fig.1 compares the numerical solution of velocity distribution for a viscous incompressible flow at different location between two faced plates and the exact solution. The result from this numerical technique converges towards the exact solution. The rate of converges increases fast as the number of elements increases. At the stationary plates, the fluid of viscosity $0.8 \mathrm{~kg}(\mathrm{~ms})^{-1}$ has zero velocity $(0 \mathrm{~m} / \mathrm{s})$, the flow velocity of the fluid is maximum $(5000 \mathrm{~m} / \mathrm{s})$, midway between the plates.

In Example 2, the Fig. 2 shows a convergence of the numerical solution of velocity distribution to the exact solution. For the fluid with viscosity 0.92 $\mathrm{kg}(\mathrm{ms})^{-1}$ and pressure gradient $12 \mathrm{~N} / \mathrm{m}^{3}$, optimal velocity $(16504.34 \mathrm{~m} / \mathrm{s})$ is noticed midway between the stationary and moving plates. The fluid particles in contact with the moving plates assumes its velocity $(400 \mathrm{~m} / \mathrm{s})$, While those in contact with the stationary plates are at 'zero velocity'. It must be noted that the numerical technique described above gives the same velocity as the 'exact value' at nodal points.

In Example 3, Fig.3 shows the numerical solution of velocity distribution of a viscous incompressible fluid of viscosity $0.4 \mathrm{~kg}(\mathrm{~ms})^{-1}$ and pressure gradient 20 $\mathrm{N} / \mathrm{m}^{3}$ at different location between the two fixed plates and the exact solution, for different number of finite elements. The results, as shown in the Figure using the technique converge fast towards the exact solution. The velocity of fluid flow assumes a maximum value of $22500 \mathrm{~m} / \mathrm{s}$ midway between both plates, and a 'zero' value at the location of the plates.

Table 1, shows the percentage difference of values between analytical solutions and solutions from our model for all the three Examples. A maximum percentage difference of $1.44 \%$ and a minimum difference of $0.0 \%$ between analytical solutions and the model were obtained. These results show that the solutions obtained using this model are highly accurate.

Conclusion: In this work we have used the finite element method (with the Lagrange interpolation function) to analyze the distribution of velocity, in viscous incompressible fluids. The model developed is highly accurate, as shown by the results obtained.

\section{REFERENCES}

Bickford, W B (1989). A First Course in the Finite Element Method, PWS Publishing Company, New York.

Burnett, D S (1987). Finite Element Analysis, from Concept to Application, Addison Wesley, New Jersey.

Demkowicz, L; Oden, J T; Rachowicz, W (1990). A new finite element method for solving compressible navier-stokes equations based on an operator splitting method and $h-p$ adaptivity. Computer Methods in Applied Mechanics and Engineering, 84(3): 275-326 S

Efunda Incorporated (2006). Fluid Mechanics: Navier-Stokes equations.

http://www.efunda.com/formulae/fluid/navierstokes.c $\underline{\mathrm{fm}}$.

Fagan, M J (1992).Finite Element Analysis, Theory and Practice, Longman Scientific and Technical, Essex, England.

Fox, RW; McDonald, AT (1996). Introduction to Fluid Mechanics. John Wiley and Sons Inc.,

New York.

Lions, P -L (1998). Mathematical Topics in Fluid Dynamics, Volume 2, Incompressible Models, Oxford Science Publications, Oxford.

Massey, B; Wardsmith, J (2001). Mechanics of Fluids. Nelson Thomas, United Kingdom

$\mathrm{Wu}, \mathrm{X}$; Jin, J( 2005). A finite element method for Stokes equation using discrete singularity expansion. Computer Methods in Applied Mechanics and Engineering 194 (1): 83-101 